\title{
Introduction to the Special Section on Functionality-Enhanced Devices
}

A $\mathrm{S}$ the semiconductor industry tries to overcome increasingly difficult technical and economic limits to scaling of CMOS transistors, switching devices are emerging with enhanced functionality. In this Special Section, we propose to focus on devices that demonstrate in-field configurable polarity, by means of electrostatic control of ambipolar conduction.

Over the past four decades, ambipolar conduction (i.e., the combined conduction behavior of $n$ - and $p$-type in the same device) was considered to be a hurdle for electronic devices. Hence, significant effort has concentrated on suppressing this mode of conduction in current CMOS technologies. However, with aggressive scaling of transistors, this is becoming increasingly difficult to achieve.

In this Special Section, we aim to gather research works examining a novel approach. In particular, by means of an additional gate, it is possible to control ambipolar conduction and to build polarity controllable devices, meaning that a single device can be in-field reconfigured to $n$-type or $p$-type.

Such functionality-enhanced devices hold the promise to build circuits with a reduced number of transistors, since they intrinsically support the XOR function. This is expected to extend the envelope of computation performance with high regularity and compactness, beyond the limits of scaling.

A very successful workshop has been organized at EPFLLausanne-Switzerland on this field. The workshop, entitled "Design with Functionality-Enhanced Devices" (FED'13http://si.epfl.ch/FED), gathered top-level research groups, with international recognition for both the design of emerging devices with controllable polarity as well as their use from a circuit and architectural perspective.

The Special Section is intended to invite the best contributions and propose for the first time a complete overview of ambipolar technology, from fabrication flows to complex architecture design, while also addressing the questions of modeling, circuit design, and associated methodologies.
Such a topic is of strategic interest for a leading journal like TNANO. Indeed, it covers a large range of fields related to advanced nanodevices. Such a multidisciplinary approach is mandatory in the research of disruptive technologies, as they generally involve a diverse set of skills and researchers from technology to architectural design. Therefore, this Special Section is intended to enforce the links within the community and to stimulate new initiatives on the promising topic of functionality-enhanced devices.

\section{PierRe-EMmanuel Gaillardon, Guest Editor EPFL-Swiss Federal Institute of Technology-Lausanne, 1015 Lausanne, Switzerland pierre-emmanuel.gaillardon@epfl.ch}

\section{Giovanni De Micheli, Guest Editor \\ EPFL-Swiss Federal Institute of Technology-Lausanne, 1015 Lausanne, Switzerland giovanni.demicheli@epfl.ch}

\section{IAN O'CONNOR, Guest Editor}

Lyon Institute of Nanotechnology-Ecole Centrale de Lyon-F-69134 Ecully, France ian.oconnor@ec-lyon.fr

\author{
SubHASISH Mitra, Guest Editor \\ Stanford University, Stanford, CA 94305, USA \\ subh@stanford.edu
}

\section{THOMAS ERNST, Guest Editor}

CEA-LETI, Minatec Campus, 38054 Grenoble, France thomas.ernst@cea.fr 